\title{
THE ANALYSIS OF LINEAR TRANSFORMATIONS
}

\section{F. J. MURRAY}

1. Introduction. If $T$ is a closed additive transformation with domain dense (abbreviated c.a.d.d.) between Hilbert spaces, there exist two resolutions of the identity, $E_{1}(\lambda)$ and $E_{2}(\lambda)$ such that $E_{2}(\lambda) T \subset T E_{1}(\lambda)$. These resolutions are defined for $0 \leqq \lambda<\infty$, and if $0<\alpha<\beta T$ is an isomorphism, when contracted to the range ${ }^{1}$ of $E_{1}(\beta)-E_{1}(\alpha)$. This is important in applications, since only under an isomorphism is the convergence of a sequence of elements equivalent to the convergence of the images. This property compensates for the lack of compactness of Hilbert space.

These resolutions also permit us to express $T$ as a denumerable sum of such isomorphisms. ${ }^{2}$ Each isomorphism in turn can be expressed in terms of the values of $T$ on an orthonormal set complete in a certain subspace. Thus $T$ is analysed into components which determine it by addition and closure. Another interesting property of these resolutions associated with $T$ is the fact that if $f$ is such that for every $g \in \mathfrak{M}_{1}(\lambda)$ (the range of $E_{1}(\lambda)$ ), $|f+g| \geqq|f|$ and if $T f$ exists then $|T f+T g| \geqq|T f|$.

When one considers a c.a.d.d. $T$ in a general reflexive Banach space, ${ }^{3}$ many properties lose their significance, but those mentioned above do not. Since they indicate a complete analysis of such transformations one is led to consider the possibility of generalizing the notion of a resolution of the identity and the association of two of these with a c.a.d.d. T.

At least five such generalizations are possible. However, the complete analysis given above cannot be carried through in general linear spaces at present because of various unsolved problems of these spaces. In the present talk, we show the dependence of this analysis upon these problems and classify the problems from this point of view. It is hoped that this will result in a more systematic development of the theory of linear spaces.

2. Projections. The difficulties appear in attempting to generalize the notion of a projection. Let us consider the usual notion of a pro-

An address delivered before the Washington meeting of the Society on May 2, 1941, by invitation of the Program Committee; received by the editors July 18, 1941.

${ }^{1}$ Cf. [11, chap. 9], or [8, \$5, pp. 312-318, in particular Theorem VI, p. 315].

2 Cf. [8, loc. cit.].

${ }^{3}$ We shall follow the notation of [1]. A reflexive space is one such that $\overline{\mathfrak{B}}=\mathfrak{B}$. 
jection in Hilbert space and endeavor to express its properties in terms which generalize to Banach spaces. This can be most readily done in terms of the range $\mathfrak{M}$ of $E$ and the set $\mathfrak{N}$, of $f^{\prime}$ 's such that $E f=0$. The following properties are present: 1 . For every $f$ in $\mathfrak{S}$, there is a $g$ in $\mathfrak{M},(g=E f)$ and an $h$ in $\mathfrak{N}$, such that $f=g+h$. 2. $\mathfrak{M} \cdot \mathfrak{N}=\{\theta\}$. 3. $\mathfrak{M}$ and $\mathfrak{N}$ are closed additive sets. ${ }^{4} 4 . h$ in $\mathfrak{N}$ and $g$ in $\mathfrak{M}$, imply $|h+g| \geqq|h|$.

In Hilbert space, given $\mathfrak{M}$, these properties ${ }^{5}$ determine a unique self-adjoint $E$, with domain $\mathfrak{S}$ and bound 1 . In suitably restricted spaces given a closed additive set, $\mathfrak{M}, 1$ and 4 will determine $\mathfrak{N} .^{6}$ Property 2 will hold but not 3 since $\mathfrak{N}$ in general will not be additive. ${ }^{7}$ One may then abandon 4 . If we are given $\mathfrak{M}$, let us call an $\mathfrak{N}$ satisfying $1,2,3$, a complement to $\mathfrak{M}$. If $\mathfrak{M}$ has a complement, $\mathfrak{N}$, the corresponding $E$ is a bounded linear transformation ${ }^{8}$ with $E^{2}=E$. Such a transformation we will call a bounded projection on $\mathfrak{M}$. Since 4 is lost, $E$ is not unique for $\mathfrak{M}$, but this is not the worst difficulty. It can be shown that there exist manifolds $\mathfrak{M}$, which do not possess bounded projections. ${ }^{9}$

One may, however, be willing to substitute for the boundedness of $E$ just closure. If we substitute for 1 , the Property $1^{\prime}$ : for a dense set of $f^{\prime} s, f=g+h, g \in \mathfrak{M}, h \in \mathfrak{N}(E f=g)$, we will call an $\mathfrak{N}$ satisfying $1^{\prime}, 2,3$, a quasi-complement to $\mathfrak{M}$. The corresponding $E$ is closed and if we have a c.a.d.d. $E$ with $E^{2}=E$, and range $\mathfrak{M}$, then $\mathfrak{M}$ has a quasicomplement. ${ }^{10}$ The following problem arises:

Problem I. In a linear space, does every $\mathfrak{M}$ possess a quasi-complement ${ }^{11}$

If $\mathfrak{M}$ does not have a complement it must be "infinite," that is,

${ }^{4}$ Properties 2 and 3 insure the uniqueness and additivity of the resolution given in 1.

${ }^{5}$ The Condition 4 implies that $\mathfrak{M}$ and $\mathfrak{R}$ are orthogonal, since $|h+t g|^{2} \geqq|h|^{2}$ for any complex $t$ implies $(h, g)=0$. Thus $E$ is the orthogonal projection on $\mathfrak{M}$. Cf. [15, Theorem 1.23 , p. 22 and Definition 2.16, p. 70$]$ or [11, chap. $2, \S 5$, Theorem VI and chap. 6, §1, Definition 1].

${ }^{6}$ Cf. Appendix I, remark preceding Lemma 4.

${ }^{7}$ In Appendix I, $\mathfrak{N}$ is the set of $h_{0}$ 's such that $F\left(h_{0}\right)=|F| \cdot\left|h_{0}\right|$ for an $F \in \mathfrak{M}^{\perp}$. We may make this correspondence more precise by letting $|F|=\left|h_{0}\right|$. Even when this correspondence is one-to-one as say in $L_{p}$ or $l_{p}(p \neq 2)$ it is not linear and $\mathfrak{R}$ the image of a linear set will not be linear in general.

3 Cf. [10, proof of Lemma 1.11].

${ }^{9} \mathrm{Cf}$. [10].

${ }^{10}$ The proof of these statements is similar to that of [10, Lemma 1.11].

${ }^{11}$ These problems are of greatest interest when $\mathfrak{B}$ is reflexive. 
infinite-dimensional and having no finite complement. We also ask:

Problem II. Do there exist Banach spaces such that every infinite manifold has no complement (quasi-complement)?

Since the Banach space $\mathfrak{B}$ and any closed additive subset $\mathfrak{M}$ in it are abelian groups, we may consider $\mathfrak{B} / \mathfrak{M}$ the quotient group of $\mathfrak{B}$ by $\mathfrak{M}$. This is the set of classes of elements of $\mathfrak{B}$, mod $\mathfrak{M}$, that is, two elements $f_{1}$ and $f_{2}$ in $\mathfrak{B}$ belong to the same class c, if $f_{1}-f_{2} \in \mathfrak{M} . \mathfrak{B} / \mathfrak{M}$ is a normed linear space if addition and scalar multiplication of classes result from the corresponding operations ${ }^{12}$ of $\mathfrak{B} \bmod \mathfrak{M}$ and $|c|=$ g.l.b. $(|f|, f \in c)$. If $\mathfrak{B}$ is reflexive, $\mathfrak{B} / \mathfrak{M}$ is complete. ${ }^{13} \mathrm{We}$ say that $\mathfrak{B}$ has been resolved abstractedly into $\mathfrak{M}$ and $\mathfrak{B} / \mathfrak{M}$.

In Hilbert space, each class c of $\mathfrak{S} / \mathfrak{M}$ contains a unique $h$ of $\mathfrak{M}^{\perp}$, with $|h|=|\mathbf{c}|$ and this correspondence $h \sim \mathbf{c}$ is linear. In a reflexive $\mathfrak{B}$, the existence for every $\mathfrak{M}$ of a complement with these properties would imply that $\mathfrak{B}$ is a Hilbert space. ${ }^{14}$ However, if additivity is dropped, we have for reflexive convex spaces an orthogonal $\mathfrak{N}$ as we have mentioned above.

3. Bounded transformations. Let us now consider the association of projections with bounded transformations. If $T$ is bounded and $\mathfrak{M}$ and $\mathfrak{N}$ are complements then $T$ takes $\mathfrak{M}$ into an additive set $T \mathfrak{M}$ and $\mathfrak{R}$ into $T \mathfrak{N}$. If $f=g+h, g \in \mathfrak{M}, h \in \mathfrak{R}, T f=T g+T h$. Thus every element $T f$ of $\Re_{T}$, the range of $T$, can be expressed as a sum of elements of $T \mathfrak{M}$ and $T \mathfrak{R}, f^{\prime}=g^{\prime}+h^{\prime}$. If $T^{-1}$ exists, we see that this resolution is unique, for $g^{\prime}=T E T^{-1} f^{\prime}$.

Thus $E$ has the corresponding operation $T E T^{-1}$ in the range space. But even in Hilbert space, this transformation may be unbounded and may even have no closed extension..$^{15}$ Thus a bounded $T$ may not preserve the relation of complementation or quasi-complementation, even when $T^{-1}$ exists.

12 The statement about the norm is the most interesting but is not particularly difficult. The closure of $\mathfrak{M}$ insures that if $|c|=0$, c contains $\theta$ and is precisely $\mathfrak{M}$, the 0 -class for addition, modulo $\mathfrak{M}$. Obviously $\left|a_{\mathbf{c}}\right|=|a| \cdot|\mathbf{c}|$ and the triangle inequality is obtained by a simple $\epsilon$-proof.

${ }^{13}$ Cf. Appendix I, Lemma 6.

${ }^{14}$ Since $h=(1-E) f$ and $|h| \leqq|f|$, we see that $1-E$ has bound 1. But $(1-E)^{*}$ $=1-E^{*}$, which would also have bound 1 . We have supposed that $\mathfrak{B}$ is reflexive and thus to every $\mathfrak{M}^{\prime} \subset \overline{\mathcal{V}}$, we can find a $\mathfrak{M} \subset \mathfrak{B}$, such that $\mathfrak{M}^{\perp}=\mathfrak{M}^{\prime}$. Hence every $\mathfrak{M}^{\prime} \subset \overline{\mathfrak{B}}$ has a projection of bound 1 on it. This implies that $\overline{\mathfrak{B}}$ is a Hilbert space and that $\overline{\mathfrak{B}}=\mathfrak{B}$ (cf. $[4]$ or $[13]$ ).

${ }^{15} \mathrm{Cf}$. Appendix II. 
However, for the special resolutions $E_{1}(\lambda)$ and $E_{2}(\lambda)$ associated with $T$ as in $\$ 1$, in Hilbert space, we do have that complementation is preserved. ${ }^{16}$ Also in Hilbert space, if $\mathfrak{M}$ is such that $T$ is an isomorphism on $\mathfrak{M}$, then a bounded $T$ preserves complementation for at least one complement. ${ }^{17}$ Thus we are interested in the $\mathfrak{M}$, for which $T$ preserves complementation.

To obtain a precise statement of our problem, let us find the exact conditions under which the equation $T E_{1}=F T$ determines a transformation $F$. Now let $g$ be given such that there is an $f$ for which $T f=g$. Then $T E_{1} f=F g$ defines a transformation $F$, if $T f_{1}=T f_{2}$ implies $T E_{1} f=T E_{2} f$ or if $T\left(f_{1}-f_{2}\right)=\theta$ implies $T E_{1}\left(f_{1}-f_{2}\right)=\theta$. Thus we will have an $F$ determined by $T$ and $E$ if $E$ projects the set of zeros of $T, \mathfrak{N}_{T}$, onto part of itself. If $\mathfrak{R}_{E_{1}} \supset \mathfrak{N}_{T}$ or $\mathfrak{N}_{E_{1}} \supset \mathfrak{N}_{T}$ then this condition is certainly fulfilled.

Now a bounded projection, $E_{1}$, will be said to be associated with a bounded $T$ (abbreviated $E_{1}$ b.A.b. $T$ ) if $\mathfrak{R}_{E_{1}} \supset \mathfrak{N}_{T}$ or $\mathfrak{N}_{E} \supset \mathfrak{N}_{T}$ and if the corresponding $F$ has a bounded extension (which will be a bounded projection $E_{2}$ ). We suppose that $\Re_{T}$ is dense in $\mathfrak{B}_{2}$.

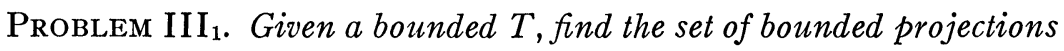
associated with it.

One can associate a closed projection with a bounded $T$ in an analogous manner ( $E_{1}$ c.A.b. $T$ ) and one has a corresponding Problem $\mathrm{III}_{2}$.

4. Closed transformations. Let us consider the similar problems for c.a.d.d. transformations. A closed transformation is characterized by the fact that the "graph," the set of pairs $\{f, T f\}$ in $\mathfrak{B}_{1} \oplus \mathfrak{B}_{2}, f$ in $\mathfrak{D}_{T}$, is closed. ${ }^{18}$

Given a projection $E_{1}$ the resolution in $\mathfrak{B}_{1}, f=g+h$, yields a resolution of the graph of a bounded $T,\{f, T f\}=\{g, T g\}+\{h, T h\}$. The graph of a bounded transformation with domain $\mathfrak{B}_{1}$ is isomorphic to $\mathfrak{B}_{1}$, and thus this latter resolution will be determined by a projection $E_{1,2}$ which is defined by the equation $E_{1,2}\{f, T f\}=\left\{E_{1} f, T E_{1} f\right\}$.

The situation is different in the case of a c.a.d.d. $T$. Since $\mathfrak{D}_{T}$ is no longer $\mathfrak{B}_{1}$, it may not include $\Re_{E_{1}}$ whether $E_{1}$ is bounded or closed. Thus if $E$ is a one-dimensional projection on an element not in $\mathfrak{D}_{T}$, the resolution cannot be imported into the graph.

This difficulty appears even in Hilbert space. When one is forced to deal with it in defining reducibility, one appends the condition:

${ }^{16}$ Cf. [8, §5, pp. 312-318].

${ }^{17}$ Cf. Appendix III.

${ }^{18}$ The graph is discussed in [16, pp. 299-301], [8, pp. 302-307], [9, pp. 88-91], and [11, chap. 4$]$. 
" $f \in \mathcal{D}_{T}$ implies $E_{1} f \in \mathfrak{D}_{T}$." When this condition is applied to either a bounded or closed $^{19} E_{1}$ in $\mathfrak{B}_{1}$, one sees that $E_{1,2}$ must be defined throughout the graph and hence is bounded. ${ }^{20}$

On the other hand, if one starts with a bounded projection $E_{1,2}$ in the graph, $E_{1,2}\{f, T f\}=\{g, T g\}$, then the equation $E_{1} f=g$ may not determine a transformation with a closed extension. ${ }^{21}$ Even if both $E_{1}$ and $E_{1,2}$ are bounded, however, the $F$ defined by the equation $T E=F T$ may not have a closed extension..$^{15}$

Definition. $A$ bounded projection, $E_{1}$, will be said to be associated with a c.a.d.d. $T,\left(E_{1}\right.$ b.A.c. $\left.T\right)$ if $\mathfrak{N}_{E} \supset \mathfrak{N}_{T}$ or $\mathfrak{N}_{E} \supset \mathfrak{N}_{T}$ and $E_{2}$ is bounded. $A$ closed projection will be said to be associated with a c.a.d.d. T (E $E_{1}$ c.A.c. $T$ ) if $\mathfrak{R}_{E} \supset \mathfrak{N}_{T}$ or $\mathfrak{N}_{E} \supset \mathfrak{N}_{T}, E_{1,2}$ is bounded and $E_{2}$ has a closed extension.

We then have Problems $\mathrm{III}_{3}$ and $\mathrm{III}_{4}$ analogous to $\mathrm{III}_{1}$ and $\mathrm{III}_{2}$, with $T$ closed rather than bounded.

5. Abstract resolutions. We have discussed the preservation by $T$ of complementation and quasi-complementation. Let us now consider abstract resolutions.

If $T$ is c.a.d.d. and $\mathfrak{M}$ is a manifold such that $\mathfrak{D}_{T}$ is dense in $\mathfrak{M}$, and $\mathfrak{M}^{\prime}$ is the closure of the set of $T f^{\prime}$ 's with $f$ in $\mathfrak{M}$, then we can consider a transformation $T$ from $\mathfrak{B}_{1} / \mathfrak{M}$ to $\mathfrak{B}_{2} / \mathfrak{M}^{\prime}$, defined as follows. Let $\mathbf{c} \in \mathfrak{B}_{1} / \mathfrak{M}$ and $f \in \mathrm{c}$. Then $T f \in \mathrm{c}^{\prime} \in \mathfrak{B}_{2} / \mathfrak{M}^{\prime}$. Now if $f_{1}$ is also in $\mathbf{c}$, $T f_{1} \in \mathbf{c}^{\prime}$, and thus $\mathbf{c}^{\prime}$ depends only on $\mathrm{c}$ and we define $T_{\mathfrak{m}} \mathbf{c}=\mathrm{c}^{\prime}$.

Now $T_{\mathfrak{M}}$ is readily seen to be additive and its domain is dense in $\mathfrak{B}_{1} / \mathfrak{M}$. In general, however, it is not closed, ${ }^{22}$ although for $T$ bounded,

${ }^{19}$ For $E_{1}$ closed the existence of $E_{1} f$ for $f$ in $\mathfrak{D}_{T}$ is implied.

${ }^{20}$ When $E_{1,2}$ is defined by the equation $E_{1,2}\{f, T f\}=\left\{E_{1} f, T E_{1} f\right\}$ and $E_{1}$ satisfies the given condition, then $E_{1,2}$ is bounded. For let $\mathfrak{M}_{1,2}$ be the set of pairs $\{f, T f\}$ for which $f$ is in $\mathfrak{M}_{1}$, the range of $E_{1}$, and let $\mathfrak{N}_{1,2}$ be the set of $\{g, T g\}$ with $g \in \mathfrak{N}_{1}$. We prove that $E_{1,2}$ is bounded by showing that $\mathfrak{M}_{1,2}$ and $\mathfrak{N}_{1,2}$ are complementary (cf. Footnote 8). The Conditions 2 and 3 on $\mathfrak{M}_{1,2}, \mathfrak{N}_{1,2}$ follow from the corresponding conditions on $\mathfrak{M}_{1}, \mathfrak{R}_{1}$ and 1 follows from the given condition on $E_{1}$.

${ }^{21}$ This is true even in Hilbert space. Let $\mathfrak{W}$ be realized as the space $\mathbb{R}_{2}$ on the interval $(0,2 \pi)$. The set of pairs, $\{\exp (i n x),-n \exp (i n x)\}, n=0, \pm 1, \pm 2, \cdots$ and $\{\exp (x)-\exp (2 \pi-x), i(\exp (x)+\exp (2 \pi-x))\}$, are orthogonal. Let $\mathfrak{I}$ denote the manifold determined by these pairs in $\mathfrak{S} \oplus \mathfrak{S}$. It is readily seen that $\mathfrak{T}$ is the graph of a transformation $T$, that is, if $\{\theta, g\}$ is in $\mathfrak{T}$, then $g=\theta$. Now if $E_{1,2}$ is the projection of $\mathfrak{T}$ onto the manifold determined by the pair $\{\exp (x)-\exp (2 \pi-x), i(\exp (x)$ $+\exp (2 \pi-x))\}$ then the corresponding $E_{1}$ has no closed extension. This is an immediate consequence of the fact that $E_{1}$ is zero on the dense set determined by the $\exp (i n x)$ for $n=0, \pm 1, \pm 2, \cdots$.

${ }^{22}$ Cf. Appendix IV. 
$T_{\mathfrak{M}}$ is bounded. On the other hand, ${ }^{23} T_{\mathfrak{M}}$ and $T$ on $\mathfrak{M}$ do not determine $T$.

But suppose $T$ and $\mathfrak{M}$ are such that if $f$ is in $\mathfrak{D}_{T}$ and in $c \in \mathfrak{B}_{1} / \mathfrak{M}$, then c contains a minimal element $h$, which is also in $\mathfrak{D}_{T}$ and $T h$ is minimal in $T_{\mathfrak{M}} \mathrm{c}$. We say then that $T$ preserves minimality relative to $\mathfrak{M}$. For reflexive convex spaces, $T_{\mathfrak{M}}$ itself is then closed. ${ }^{24}$

We will say that the abstract resolution of a manifold $\mathfrak{M}$ is associated with a closed $T$ if $T$ preserves minimality relative to $\mathfrak{M}$.

In Hilbert space, the resolutions associated with $T$ are precisely those which break $T$, that is, those which reduce ${ }^{25}\left(T^{*} T\right)^{1 / 2}$. Thus this criterion determines the manifolds more precisely than the previous ones.

Problem IV $\mathrm{IV}_{1}\left(\mathrm{IV}_{2}\right)$. Given a bounded $T$ (c.a.d.d. T), find the abstract resolutions associated with it.

In Hilbert space the answer to these problems is known. In general linear spaces the answer is not so interesting but we will consider other generalizations of the manifolds which reduce $\left(T^{*} T\right)^{1 / 2}$.

Notice that if the abstract resolution with respect to $\mathfrak{M}$ is associated with $T$, the latter can be regarded as resolved into linear components of $T$ on $\mathfrak{M}$ and $T_{\mathfrak{M}}$. This notion is particularly interesting for $\mathfrak{M}=\mathfrak{N}_{T}$, whose abstract resolution is associated with $T$.

6. Bases. We now consider the general problem of reducing $T$ to linear components, rather than to the special case in which there are only two components. One such resolution is given by any basis for the graph and thus we may consider the basis problem.

Banach defines a basis $\left[1\right.$, p. 110] as a sequence of elements $\left\{f_{i}\right\}$ such that to each $f \in \mathfrak{B}$, there exists a unique sequence of numbers $\eta_{i}$ such that $f=\sum_{i=1}^{\infty} \eta_{i} f_{i}$. This definition implies that $\eta_{i}=F_{i}(f)$ is an additive linear functional of $f[1$, p. 111].

Problem V. Does every separable Banach space have a basis?

The graph of a bounded transformation is isomorphic to the domain space and thus a basis in $\mathfrak{B}_{1}$ determines a basis in $\mathfrak{T}$, and hence a resolution into irreducible linear components. For a closed transformation one must consider the graph directly.

${ }^{23}$ A two-dimensional example will make this clear. Let $T_{\alpha}$ be defined by the equation $T_{\alpha}\{x, y\}=\{x+\alpha y, y\}$. If $\mathfrak{M}$ is the set of vectors $\{x, 0\}$, one sees that $T_{\alpha}$ on $\mathfrak{M}$ and $T_{\mathfrak{R}}$ do not determine $T_{\alpha}$.

${ }^{24}$ Cf. Appendix V.

${ }^{25}$ Cf. [8, §5, pp. 312-318]. 
For a bounded $T$, given a subsequence of a basis $\left\{f_{i_{\alpha}}\right\}$ such that $\sum \eta_{i \alpha} f_{i \alpha}$ converges for every $f$ one would consider the corresponding projection $E f=\sum \eta_{i_{\alpha}} f_{i_{\alpha}}$. However, it is not known whether every infinite subsequence of a basis series is convergent. When every such subsequence is convergent, we will call the basis an absolute basis. The existence question for these is also important and we shall call it $\mathrm{V}_{\mathbf{2}}$. We also have this problem:

Problem VI. Is every basis an absolute basis?

7. Resolutions of the identity. A basis can be regarded as a special case of a resolution of the identity. One way of defining the latter notion is given by Lorch [5, Definition 4, p. 226]. This is the most restrictive way. A family of projections $E(\lambda)$ is said to constitute a resolution of the identity: 1 . if 0 and 1 are in the family; 2 . if $\lambda \geqq \mu$, $E(\lambda) E(\mu)=E(\mu)=E(\mu) E(\lambda) ; 3$. if there exists a constant $K$, such that for any real numbers $a_{i}, b_{i},(i=1, \cdots, n)$ with $a_{1} \leqq b_{1} \leqq \cdots \leqq a_{i} \leqq b_{i}$ $\leqq \cdots \leqq a_{n}, b_{n}$ and for any complex numbers $\mu_{i}$ with $\left|\mu_{i}\right| \leqq 1$, the bound of the operator $\sum_{\alpha=1}^{n} \mu_{\alpha}\left(E\left(b_{\alpha}\right)-E\left(a_{\alpha}\right)\right)$ does not exceed $K$.

One may point out that if one replaces the condition $\left|\mu_{i}\right| \leqq 1$ by $\left|\mu_{i}\right|=1$, the new Condition $3^{\prime}$ is equivalent ${ }^{26}$ to 3 . This type of resolution will be referred to as an absolutely bounded resolution of the identity. Other definitions of a resolution of the identity may be obtained by replacing the Condition 3 or $3^{\prime}$ by any one of the following: $3^{\prime \prime}$. There exists a $K$ such that for every $\lambda$, the bound of $E(\lambda)$ is less than or equal to $K .3^{\prime \prime \prime}$. The $E(\lambda)$ exist and are bounded except possibly on a denumerable, nowhere dense set of points. $3^{\text {IV }}$. Each $E(\lambda)$ is closed. The corresponding resolutions will be termed, respectively, bounded, essentially bounded, and closed. To make our existence problem definite we also introduce the definition.

If $f \in \mathfrak{B}$, and $a_{1}, b_{1}, \cdots, a_{n}, b_{n}$ are as in Condition 3 above, let $\mathfrak{A}_{f}$ denote the set of $g$ 's for which there are $\lambda_{\alpha}$ 's such that $g=\sum_{\alpha=1}^{n} \lambda_{\alpha}\left(E\left(b_{\alpha}\right)\right.$ $\left.-E\left(a_{\alpha}\right)\right) f$. If there is an $f$, such that $\mathfrak{A}_{f}$ is dense, then we say that the resolution of the identity has a simple spectrum.

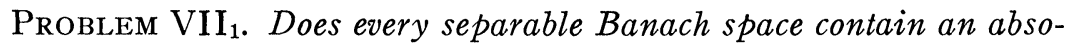
lutely bounded resolution of the identity with a simple spectrum?

For bounded, essentially bounded or closed resolutions, we have the corresponding Problems $\mathrm{VII}_{2}, \mathrm{VII}_{3}, \mathrm{VII}_{4}$.

20 A proof of the equivalence of 3 and $3^{\prime}$ can be outlined as follows: If $|\mu| \leqq 1$ ( $\mu$ real), then $|f+\mu g| \leqq \max (|f+g|,|f-g|)$. This can be used to show that for a given set of $\mu_{i}$ 's and an $f$, we can find a set of $\epsilon_{i}$ 's with $\left|\epsilon_{i}\right|=1$, such that $\mid \sum \mu_{i}\left(E\left(b_{i}\right)\right.$ $\left.-E\left(a_{i}\right)\right) f|\leqq| \sum \epsilon_{i}\left(E\left(b_{i}\right)-E\left(a_{i}\right)\right) f \mid$. This can be used to show that the least $K$ of $3^{\prime}$ is greater than or equal to the least $K$ of 3 . 
Now corresponding to a basis, we can find a bounded resolution of the identity. ${ }^{27} \mathrm{~A}$ basis is absolute if and only if the corresponding resolution is absolutely bounded. ${ }^{28}$ Thus we may generalize Problem $\mathrm{VI}_{1}$ to the following problem:

\section{Problem $\mathrm{VI}_{2}$. Is every bounded resolution of the identity absolutely bounded?}

8. Resolutions and transformations. We need to consider only resolutions such that $E_{1}(\lambda)=0$, if $\lambda<0$. A resolution $E(\lambda)$ will be said to be associated with a transformation $T: 1$. if $E(0)$ is the manifold of zeros of $T$. 2. If the resolution $E(\lambda)$ is absolutely bounded, bounded or essentially bounded and $E(\lambda)$ is bounded, then $T$ and $E(\lambda)$ are associated as in Problems $\mathrm{III}_{1}$ or $\mathrm{III}_{3}$. If the resolution is considered simply as closed, then $T$ and $E(\lambda)$ are associated as in Problems III $_{2}$ or $\mathrm{III}_{4}$. 3. If the resolution $E(\lambda)$ is absolutely bounded, bounded or closed and $0<\alpha<\beta$ then $T$ is an isomorphism on the range of $E(\beta)-E(\alpha)$. If $E(\lambda)$ is essentially bounded and $0<\alpha<\beta$ and there is no singular point between $\alpha$ and $\beta$, then $T$ is an isomorphism on the range of $E(\beta)-E(\alpha)$.

A special case is that in which $T$ takes the range of $E(\beta)-E(\alpha)$ into itself. (Here certain of the above restrictions may be ignored.) Lorch has shown that if $T$ is associated in this way with an absolutely bounded resolution, then one has an operational calculus for $T$ [5] and the converse of this is quite simple.

On the other hand one can show that there exist bounded transformations which are not associated with any absolutely bounded or

${ }^{27}$ Given a basis, $x_{1}, x_{2}, \cdots$, with the corresponding functionals, $f_{1}, f_{2} \ldots$, a resolution of the identity can be obtained as follows: Let $\left\{\lambda_{i}\right\}$ be a sequence of positive numbers such that $0<\lambda_{1}<\lambda_{2}<\cdots<1$, and $\lim _{i \rightarrow \infty} \lambda_{i}=1$. Let $n(\lambda)=0$, for $\lambda<\lambda_{1}$, $n(\lambda)=n$ if $\lambda_{n} \leqq \lambda<\lambda_{n+1}$ and for $\lambda \geqq 1, n(\lambda)=\infty$. Then for $n(\lambda)=n$ let $E(\lambda) y=\sum_{i=1}^{n} f_{i}(y) x_{i}$ Since the partial sums of a convergent biorthogonal series are uniformly bounded, the $E(\lambda)$ 's are readily proved to constitute a resolution of the identity.

${ }^{28}$ In the construction of the preceding footnote given any finite subset $x_{1}^{\prime}, \ldots, x_{m}^{\prime}$ of the $x_{1}, x_{2}, \cdots$ and corresponding functionals, $f_{1}^{\prime}, \cdots, f_{m}^{\prime}$, we can find a set $a_{i}, b_{i}$ such that $\sum\left(E\left(b_{i}\right)-E\left(a_{i}\right)\right) y=\sum_{i=1}^{m} f_{i}^{\prime}(y) x_{i}^{\prime}$. Thus if the corresponding resolution is absolutely bounded, the partial sums of the subsequences are also uniformly bounded and the basis is absolute. The proof of the converse is longer but not essentially more difficult. In $\sum \mu_{i}\left(E\left(b_{i}\right)-E\left(a_{i}\right)\right)$ we consider the real and imaginary parts of the sum. In either part, we can replace the corresponding part of $\mu_{i}$ by either +1 or -1 and at most increase the bound. Thus it can be shown that the bound of the given sum does not exceed the sum of the bounds of four sums in the form $\sum E\left(b_{i}\right)-E\left(a_{i}\right)$. The uniform bound of the latter is of course determined by the uniform bounds of the arbitrary partial sums if the given basis is absolute (cf. [6, pp. 564-566]). 
even bounded resolution of the identity. ${ }^{29}$ However we may still ask this question:

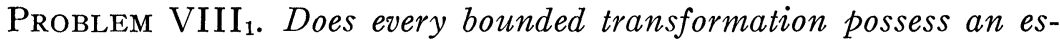
sentially bounded resolution? ${ }^{30}$

A similar Problem VIII $_{2}$ arises when we simply ask for a closed resolution. A closed transformation determines a bounded transformation from the graph to the domain space $A\{f, T f\}=f$. It is readily seen that if $A$ possesses a resolution, $T$ will also. Thus we need not consider closed transformations.

9. Abstract resolutions and transformations. One may also attempt to define the notion of association, relative to the abstract resolutions on a family $\mathfrak{M}(\lambda)$ of manifolds. Here again we need only concern ourselves with bounded $T$ 's and a tentative definition is: 1 . M( $(0)$ is $\mathfrak{N}_{T}$. 2. $\mathfrak{M}(\lambda) \subset \mathfrak{M}(\mu)$ for $\lambda<\mu .3^{\prime} . \mathfrak{M}(\lambda)$ is associated with $T$ as in Problem IV.

What is desired here is a generalization of the notion of the resolution of the absolute value of the transformation and a consideration of examples with finite-dimensional domain spaces shows that here this definition does not yield the desired result. Nevertheless in these cases the usual "choosing the maximum" construction does yield a family $\mathfrak{M}(\lambda)$ for $0<\lambda<C, C$ being the bound of $T$ with the Properties 1,2 and 3. $T$ has bound $\lambda$ on $\mathfrak{M}(\lambda)$, and 4 . The family $\mathfrak{M}(\lambda)$ is maximal, that is, if $f \in \mathfrak{M}(\lambda)$, there exists a $\lambda^{\prime} \geqq \lambda$, for which $f \in \mathfrak{M}\left(\lambda^{\prime}\right)$ implies that 2 or 3 is false.

For our general discussion, we introduce the following definitions: $\mathfrak{M}$ will be said to be an over- $\lambda$-manifold if $f \in \mathfrak{M}$ implies $|T f| \geqq \lambda|f|$. $\mathfrak{N} \subset \overline{\mathfrak{B}}$ will be said to be a coverover- $\lambda$-manifold if $|T f| \geqq \lambda|f|$ implies that there is an $F$ in $\mathfrak{N}$ with $F(f) \neq 0$. Similarly we define an under- $\lambda$ manifold and coverunder- $\lambda$-manifold.

In Hilbert space there are manifolds which are both over- $\lambda$-manifolds and coverover- $\lambda$-manifolds. Since in more general spaces, these are in different spaces, one can't expect this result to generalize readily. However it may happen that $\mathfrak{M}(\lambda)$, for $\lambda<0$, may have a complement or quasi-complement which is an over- $\lambda^{\prime}$-manifold for $\lambda^{\prime}>0$. We say then that the resolution $\mathfrak{M}(\lambda)$ is conjugated and thus we have this problem:

${ }^{29}$ Cf. Appendix VI.

${ }^{30}$ If an e.b. resolution is associated with $T$, it is not in general unique. However if the answer to Problem VII $\mathrm{VI}_{1}$ yes and one e.b. resolution exists for $T$, then there is also one with simple spectrum. In general, each Problem VIII can be referred to the projection situation for $\mathfrak{N}_{T}$ and the answers to Problems V or VII. 
Problem IX. Do there exist transformations $T$ which do not possess a conjugated manifold family?

One can establish without great difficulty, the existence of a family of manifolds $\mathfrak{M}(\lambda)$ with the Properties ${ }^{31} 1,2,3$, 4. The definitions yield that if $\mathfrak{M}$ is a maximal under- $\lambda$-manifold, $\mathfrak{M}^{\perp}$ is a minimal cover over- $\lambda$-manifold.

10. Comment. The above discussion shows clearly the approach to the analysis of a specific transformation $T$. For instance, let us consider the problem of determining the projections $E$, which are associated with a given $T$ as in Problem III $_{1}$. Suppose $T^{-1}$ exists. This association is equivalent to the statement "TET ${ }^{-1}$ is bounded." Suppose the bound is $k$. Let $\mathfrak{M}$ be an under- $\lambda$-manifold for $T$ and an over$\sigma$-manifold for $E$. Then $E M$ must be an under- $k \lambda / \sigma$-manifold for $T$. For a bounded $E$ a similar condition is sufficient. Thus the problem can be referred to the $\lambda$-manifold.

Problem X. Given $T$, find the various $\lambda$-manifolds.

While this discussion does not present an exhaustive list of the problems one meets in this field, the others are apparent from the given considerations.

\section{Appendix I}

We establish certain results which are essential in the theory of abstract resolutions. We consider a vector space $\mathfrak{B}$ and $\mathfrak{M}$ a closed linear set in $\mathfrak{B}$. Let $\mathfrak{B} / \mathfrak{M}$ be as in $\S 2$ and $\mathfrak{B}_{\mathfrak{M}}$ denote the conjugate space to $\mathfrak{B} / \mathfrak{M}$.

\section{LEMma 1. $\mathfrak{M}^{\perp}$ and $\mathfrak{B}_{\mathfrak{M}}$ are isomorphic.}

Let us correspond to a functional $G$ in $\mathfrak{B}_{\mathfrak{M}}$, a functional $F$ on $\mathfrak{B}$ defined by means of the equation $F(f)=G(\mathbf{c})$ if $f \in$ c. One can readily show that $F$ is linear with $|F|=|G|$ and $F \in \mathfrak{M}^{\perp}$. On the other hand, given an $F \in \mathfrak{M}^{\perp}$, we see that $F(f)$ is a function of the class, modulo $\mathfrak{M}$, of $f$, that is, $F(f)=G(\mathbf{c}), f \in c$, for some $G \in \mathfrak{B}_{\mathfrak{M}}$. Thus the correspond-

${ }^{31}$ Suppose that the bound of $T$ is 1 . To obtain the manifolds $\mathfrak{M}(\lambda)$, we proceed as follows: It is easy to prove that if $\mathfrak{M}$ is under- $\lambda$, there is a maximal under- $\lambda$ manifold which contains it. Thus we may take $\mathfrak{M}(1 / 2)$ as a maximal under- $1 / 2$ manifold which contains $\mathfrak{N}_{T}, \mathfrak{M}(3 / 4)$ as a maximal under-3/4-manifold which contains $\mathfrak{M}(1 / 2), \mathfrak{M}(1 / 4)$ as a maximal under-1/4-manifold contained in $\mathfrak{M}(1 / 2)$ and containing $\mathfrak{N}_{T}$, and so on. For other values of $\lambda$ between 0 and $1, \mathfrak{M}(\lambda)$ is the intersection of the manifolds defined for larger values of $\lambda$. The resulting family can be shown to have the Properties 1, 2, 3, 4 . 
ence, $F \sim G$ is one-to-one between $\mathfrak{M}^{\perp}$ and $\mathfrak{B}_{\mathfrak{M}}$ and metric preserving. Finally one observes that it is additive and hence $\mathfrak{B}_{\mathfrak{M}}$ and $\mathfrak{M}^{\perp}$ are isomorphic.

Lemma 2. Suppose $\mathfrak{B}$ is reflexive. Given an element $c_{0} \in \mathfrak{B} / \mathfrak{M}$, there exists an $h_{0} \in \mathfrak{B}$ such that if $F \in \mathfrak{M}^{\perp}$ corresponds to $G \in \mathfrak{B}_{\mathfrak{M}}$ as in Lemma 1 , then $F\left(h_{0}\right)=G\left(\mathrm{c}_{0}\right)$ and $\left|h_{0}\right|=\left|\mathrm{c}_{0}\right|$.

Each $\mathbf{c}_{0}$ in $\mathfrak{B} / \mathfrak{M}$ determines a linear functional $K_{0}(G)$ of the $G \in \mathfrak{B}_{\mathfrak{M}}$, with $K_{0}(G)=G\left(\mathbf{c}_{0}\right)$. Correspondingly, we have an $H_{0}$ defined on $\mathfrak{M}^{\perp}$ such that $H_{0}(F)=K_{0}(G)$. Now $H_{0}$ has an extension $H$ defined on $\overline{\mathfrak{B}}$ with $\left|H_{0}\right|=|H|$ and such that for $F \in \mathfrak{M}^{\perp}, H_{0}(F)=H(F)$ [1, corollary p. 29]. (For the extension giving complex homogeneity refer to [2]. A similar proof is given in [9] for $\mathfrak{B}=L_{p}$ which can be readily generalized.) Since $\mathfrak{B}$ is reflexive, there exists an $h_{0} \in \mathfrak{B}$ such that $F\left(h_{0}\right)=H(F)$ for all $F \in \overline{\mathfrak{B}}$. Thus if $F \sim G$ as in Lemma $1, G\left(c_{0}\right)=K_{0}(G)$ $=H_{0}(F)=H(F)=F\left(h_{0}\right)$ and $\left|\mathbf{c}_{0}\right|=\left|K_{0}\right|=\left|H_{0}\right|=|H|=\left|h_{0}\right|$.

Lemma 3. The $h_{0}$ of Lemma 2 is in $\mathrm{c}_{0}$ and minimal in $\mathrm{c}_{0}$, that is, $h_{0}$ is such that $\left|h_{0}+g\right| \geqq\left|h_{0}\right|$ for every $g$ in $\mathfrak{M}$.

If $f \in c_{0}$ and $F \in \mathfrak{M}^{\perp}$, we have a $G \in \mathfrak{B}_{\mathfrak{M}}$, such that $F(f)=G\left(c_{0}\right)$ $=F\left(h_{0}\right)$. But $F(f)=F\left(h_{0}\right)$ or $F\left(f-h_{0}\right)=0$ for every $F \in \mathfrak{M}^{\perp}$ implies $f-h_{0} \in\left(\mathfrak{M}^{\perp}\right)^{\perp}$. Now $\left(\mathfrak{M}^{\perp}\right)^{\perp}=\mathfrak{M}$ since $\mathfrak{B}$ is reflexive. (Cf. $[9$, proof of Theorem 1.3 , p. 85].) Thus $f-h_{0} \in \mathfrak{M}$, which implies, since $f \in \mathrm{c}_{0}$, that $h_{0} \in c_{0}$. Since $\left|h_{0}\right|=\left|c_{0}\right|,\left|h_{0}\right| \leqq|f|$ for every $f \in c_{0}$. Furthermore $h_{0}+g$ is in $\mathbf{c}_{0}$ for $g \in \mathfrak{M}$ and thus we have shown the second statement.

A space is said to be strictly convex, if $|f+g|<|f|+|g|$ when $f$ and $g$ are not linearly dependent.

Lemma 4. The minimal $h_{0}$ is unique, if $\mathfrak{B}$ is strictly convex.

If $\left|\mathbf{c}_{0}\right|=0$, the minimal element is precisely $\theta$. Suppose $\left|\mathbf{c}_{0}\right| \neq 0$ and that $h_{0}$ and $h$ are distinct minimal elements. It is readily seen that $h_{0}$ and $h$ are linearly independent and that also $\frac{1}{2}\left(h_{0}+h\right)$ is in $c_{0}$. Since strict convexity implies that $\left|\frac{1}{2}\left(h_{0}+h\right)\right|<\frac{1}{2}\left|h_{0}\right|+\frac{1}{2}|h|=\left|c_{0}\right|$, we have a contradiction and only one minimal element is possible.

J. Clarkson has shown that if $\mathfrak{B}$ is uniformly convex (cf. [3, Definition 1, p. 396]) then each $c_{0}$ contains a minimal element $h_{0}$. This result is unpublished and is less general than that given above (cf. [8] or [12]). In this connection one should also mention the result of Clarkson [3, p. 413], that any separable Banach space can be assigned an equivalent norm, which is strictly convex.

In the cases in which the minimal element is unique, conditions 1 and 4 of $\$ 2$, determine precisely the set $\mathfrak{N}$ of minimal elements. 
LEMma 5. The set of elements $h_{0}$, minimal relative to $\mathfrak{M}$, is the set of elements $h$ for which there is an $F \neq 0$ and $\in \mathfrak{M}^{\perp}$, such that $|F| \cdot|h|$ $=F(h)$.

Suppose $F \neq 0$ and $\in \mathfrak{M}^{\perp}$ and $h$ are such that $|F| \cdot|h|=F(h)$. Thus if $g \in \mathfrak{M}, F(g)=0$ and $|F| \cdot|h+g| \geqq F(h+g)=F(h)=|F| \cdot|h|$. Hence $|h+g| \geqq|h|$ and $h$ is minimal relative to $\mathfrak{M}$. On the other hand suppose $h_{0}$ is minimal relative to $\mathfrak{M}$ and $h_{0} \in c_{0}$. There is a $G_{0} \in \mathfrak{B}_{\mathfrak{M}}$, such that $G_{0} \neq 0$ and $\left|G_{0}\right| \cdot\left|\mathbf{c}_{0}\right|=G_{0}\left(\mathbf{c}_{0}\right)$. (Cf. [1, chap. 4, \$2, Theorem 3, p. 55].) By Lemma 1, we have an $F \in \mathfrak{M}^{\perp}$, with $|F|=\left|G_{0}\right|$ and $F\left(h_{0}\right)=G_{0}\left(\mathbf{c}_{0}\right)=\left|G_{0}\right| \cdot\left|\mathbf{c}_{0}\right|=|F| \cdot|h|$. Thus $h_{0}$ is an $h$ for which there is an $F \in \mathfrak{M}^{\perp}$ such that $F\left(h_{0}\right)=|F| \cdot\left|h_{0}\right|$. This completes the proof of the lemma.

Another result is this lemma:

LEMma 6. If $\mathfrak{B}$ is reflexive, then $\mathfrak{B} / \mathfrak{M}$ has the same property and in particular is complete.

Proof. Let $t(G)$ be a linear functional on the set of linear functionals on $\mathfrak{B} / \mathfrak{M}$. Correspondingly there is a functional $t^{\prime}(F)$ on $\mathfrak{M}^{\perp}$. This can be extended to $\overline{\mathfrak{B}}$ and hence $t^{\prime}(F)=F(k)$ for some $k$ of $\mathfrak{B}$. If $\mathrm{c}$ is the class of $k$, we have $t(G)=t^{\prime}(F)=F(k)=G(\mathrm{c})$. Thus $\mathfrak{B} / \mathfrak{M}$ is the set of linear functionals on a complete space and is readily proved to be complete.

\section{APPENDIX II}

Let $\mathfrak{M}$ and $\mathfrak{N}$ be orthogonal complements in $\mathfrak{S}$. Let $T$ be bounded, $\Re_{T}$ dense and such that $T^{-1}$ exists. We shall show that there exist $T$ 's, $\mathfrak{M}$ 's and $\mathfrak{N}$ 's such that the closure $\mathfrak{M}^{\prime}$ and $\mathfrak{N}^{\prime}$ of $T \mathfrak{M}$ and $T \mathfrak{N}$ are neither complementary nor quasi-complementary and if $E$ is the projection on $\mathfrak{M}, T E T^{-1}$ does not have a closed extension.

Suppose we have a complete orthonormal set $\phi_{0}, \phi_{1}, \cdots$ and a sequence of numbers $\lambda_{i}>0$, with $\sum_{i=0}^{\infty} \lambda_{i}<\infty$. Let $\beta_{i}=\left(1-\lambda_{i}^{2}\right)^{1 / 2}$. Define a transformation $T^{\prime \prime}$ by means of the equation $T^{\prime \prime} \phi_{i}=\lambda_{i}\left(\lambda_{i} \phi_{i+1}+\beta_{i} \phi_{0}\right)$ and let $T^{\prime}$ be defined as the least additive extension of $T^{\prime \prime}$. (Cf. [15, p. 45, Theorem 2.10] or [11, Definition 4, p. 32].)

$T^{\prime}$ is bounded. For the domain of $T^{\prime}$ consists of those $f^{\prime} \mathrm{s}$ in the form $\sum_{i=0}^{n} a_{i} \phi_{i}$ and each $a_{i}$ is such that $\left|a_{i}\right| \leqq|f|$. Then $\left|T^{\prime} f\right|$ $=\left|\sum_{i=0}^{n} a_{i} T \phi_{i}\right| \leqq \sum_{i=0}^{n}\left|a_{i}\right|\left|T \phi_{i}\right| \leqq \sum_{i=0}^{n}|f| \cdot \lambda_{i} \leqq|f| \sum\left|\lambda_{i}\right| \leqq C|f|$. Since $T^{\prime}$ is bounded, it possesses a bounded extension, $T$ with domain the full space. (Cf. $[15$, p. 57 , Theorem 2.23$]$ or $[11$, p. 10 , Theorem II].)

For $T$, we have that $T f=\theta$ implies $f=\theta$. For let $f=\sum_{i=0}^{\infty} a_{i} \phi_{i}$. Then $T f=\sum_{i=0}^{\infty} a_{i} \lambda_{i}\left(\lambda_{i} \phi_{i+1}+\beta_{i} \phi_{0}\right)=\left(\sum_{i=0}^{\infty} \lambda_{i} \beta_{i} a_{i}\right) \phi_{0}+\sum_{i=1}^{\infty} \lambda_{i}^{2} a_{i-1} \phi_{i}$. Thus $T f$ 
$=\theta$ implies $\lambda_{i}^{2} a_{i-1}=0$ for $i=1,2, \cdots$ or $a_{i}=0$ for $i=0,1, \cdots$. Hence $f=\theta$ and $T$ has an inverse.

$\Re_{T}$ is dense, for $\Re_{T}$ contains $\lambda_{i} \phi_{i+1}+\beta_{i} \phi_{0}$ and since $\lambda_{i} \rightarrow 0, \beta_{i} \rightarrow 1$, the closure $\left[\Re_{T}\right]$ must contain $\phi_{0}$. Subtracting $\beta_{i} \phi_{0}$, we can show that $\left[\Re_{T}\right]$ also contains $\phi_{i+1}$ for $i=0,1, \cdots$. Hence $\left[\Re_{T}\right]$ contains a complete orthonormal set and is the full space. (Cf. [11, p. 21].)

Let $\mathfrak{M}$ be the closed linear manifold determined by the $\phi_{i}$ 's with even subscripts, $\mathfrak{N}$ by those with odd subscripts. $\mathfrak{M}$ and $\mathfrak{N}$ are orthogonal complements. It is readily seen that $[T \mathfrak{M}]$ and $[T \mathfrak{N}]$ both contain $\phi_{0}$ and thus are not even quasi-complementary.

If $E$ is the orthogonal projection on $\mathfrak{M}, T E T^{-1}=F$ does not have a closed extension. For let $f_{k}=\lambda_{2 k} \phi_{2 k+1}+\beta_{2 k} \phi_{0}$, then $F f=f$ and $f_{k} \rightarrow \phi_{0}$. Hence if $F$ has a closed extension $[F]$ then $[F] \phi_{0}$ would exist and equal $\phi_{0}$. On the other hand, if $g_{k}=\lambda_{2 k+1} \phi_{2 k+2}+\beta_{2 k} \phi_{0}$, we have $F g_{k}=\theta$ and $g_{k} \rightarrow \phi_{0}$. Thus if $[F]$ exists, we would have $[F] \phi_{0}=\theta$. This contradiction shows that $[F]$ does not exist.

While the above argument is given only for Hilbert space, it can be readily extended to any space having a basis, $\phi_{0}, \phi_{1}, \cdots$ for which there is a $k$ such that $f=\sum_{i=0}^{\infty} a_{i} \phi_{i}$ implies $\left|a_{i}\right| \leqq k|f|$.

\section{Appendix III}

Let $T$ be a bounded transformation from $\mathfrak{S}_{1}$ to $\mathfrak{S}_{2}$ or in $\mathfrak{E}$ and let $\mathfrak{M}$ be a closed linear manifold such that $T$ is an isomorphism on $\mathfrak{M}$. We shall show that $T$ preserves complementation for at least one complement to $\mathfrak{M}$.

Let $W H=T$ be the canonical resolution of $T$ where $H$ is self-adjoint definite, $W$ partially isometric. (Cf. [16, Theorem 7, p. 307] or [8, Theorem 1.24, p. 312] or $[11$, chap. 9, p. 95].) Let $H$ have the form $\int_{0}^{\infty} \lambda d E_{1}(\lambda)$. We suppose, as we may, that $\mathfrak{S}_{2}$ is precisely the closure of the range of $T$.

LeмmA 1. There exists a c.a.d.d. transformation $T^{\prime \prime}$ from $\mathfrak{S}_{2}$ to $\mathfrak{S}_{1}$, whose domain is $\Re_{T}$ and whose range is the range of $1-E_{1}(0)$ and which is such that $T T^{\prime \prime} \subset 1, T^{\prime \prime} T=1-E_{1}(0)$.

If $h \in \Re_{T}$, there is a unique $g$ in $\Re_{1-E_{1}(0)}$ such that $T g=h . h \in \Re_{T}$ implies that there is an $f$ such that $T f=h$. Let $g=\left(1-E_{1}(0)\right) f$. $T E_{1}(0)=0$ implies $T f=T g=h$. This $g$ is uniquely determined by $h$. for if $T g_{1}=h=T g_{2}$, we have that $T\left(g_{1}-g_{2}\right)=\theta$. Thus $g_{1}-g_{2}$ is in the range of $E_{1}(0)$ and $1-E_{1}(0)$ and hence is $\theta$. Let $T^{\prime \prime} h=g$,

From the above, we see that if $h$ is in $\Re_{T}$, then $T T^{\prime \prime} h=T g=h$ and thus $T T^{\prime \prime} \subset 1$. For $g$ in $\Re_{1-E_{1}(0)}, T^{\prime \prime} T g=T h=g$ and if $f$ is in 
$\mathfrak{N}_{T}=\mathfrak{N}_{1-E_{1}(0)}, T^{\prime \prime} T f=T^{\prime \prime} \theta=\theta$. The linearity of $T^{\prime \prime} T$ yields that $T^{\prime \prime} T=1-E_{1}(0)$.

Lemma 2. Let $F$ be a projection $\mathfrak{M}=\mathfrak{R}_{F}, \mathfrak{N}=\mathfrak{N}_{F}, \mathfrak{N} \supset \mathfrak{N}_{T}$. If $T F T^{\prime \prime}$ is bounded, its closure is a projection and $\mathfrak{M}^{\prime}$ and $\mathfrak{N}$ 'are complementary.

$F_{2}=T F T^{\prime \prime}$ has domain $\Re_{T}$ which is dense. Thus $\left[F_{2}\right]$ has domain $\mathfrak{S}_{2}$. Since $\mathfrak{N}_{F} \supset \mathfrak{N}_{1}, F E_{1}(0)=0$ and $F\left(1-E_{1}(0)\right)=F$. Thus $T F T^{\prime \prime} T F T^{\prime \prime}$ $=T F\left(1-E_{1}(0)\right) F T=T F^{2} T^{\prime \prime}=T F T^{\prime \prime}$. Thus $F_{2} F_{2}=F_{2}$. This yields $\left[F_{2}\right]^{2}=\left[F_{2}\right]$, since $F$ is bounded. Thus $\left[F_{2}\right]$ is a projection.

$\mathfrak{R}_{F_{2}}=T \mathfrak{M}$ for $F_{2}=T F T^{\prime \prime}$ implies $\mathfrak{R}_{F_{2}} \subset T \mathfrak{M}$ while if $g \in T \mathfrak{M}, g=T f$, $f \in \mathfrak{M}$ and

$$
F_{2} g=T F T^{\prime \prime} T f=T F(1-E(0)) f=T F f=T f=g .
$$

Thus for $g \in T \mathfrak{M}, F_{2} g=g$. Thus $\mathfrak{R}_{F_{2}}=T \mathfrak{M}$ and $\mathfrak{R}_{\left[F_{2}\right]}=\mathfrak{M}^{\prime}$.

A similar discussion will show $\mathfrak{N}_{F_{2}}=T \mathfrak{N}$ since $\mathfrak{N} \supset \mathfrak{N}_{T}$. Now if $g \in \mathfrak{R}_{T}=\mathfrak{D}_{F_{2}}$, then $g=h_{1}+h_{2}, h_{1} \in T \mathfrak{M}, h_{2} \in T \mathfrak{N}$ and $F_{2} g=h_{1}$. Now if $\left[F_{2}\right] f=\theta$, there must be a sequence of such $g$ 's approaching $f$ with the corresponding $h_{1}$ 's approaching $\theta$. Thus $f$ is also the limit of the $h_{2}$ 's and hence is in $\mathfrak{N}^{\prime}$. Thus $\mathfrak{N}^{\prime}=\mathfrak{N}_{\left[F_{2}\right]}$.

Since $F$ is bounded these facts show that $\mathfrak{M}^{\prime}$ and $\mathfrak{N}^{\prime}$ are complementary.

LEMmA 3. Let $T$ be an isomorphism on $\mathfrak{M}$. Then there exists a $\lambda_{0}>0$, such that $1-E_{1}\left(\lambda_{0}\right)$ when contracted to $\mathfrak{M}$ is also an isomorphism.

Since $T$ is an isomorphism on $\mathfrak{M}$, there exists a constant $C$ such that for $f \in \mathfrak{M},|T f| \geqq C|f|$. (Cf. [1, chap. 3, Theorem 5, p. 41] or [11, p. 50, Theorem IV].) This may be written

$$
\left|T E_{1}(C / 2) f+T\left(1-E_{1}(C / 2)\right) f\right|^{2} \geqq C_{1}^{2}|f|^{2} .
$$

Since $T$ preserves orthogonality for manifolds which reduce $H[8$, $\$ 5$, Definition 1, p. 312 and Theorem IV, p. 313], we get

$$
\left|T E_{1}(C / 2) f\right|^{2}+\left|T\left(1-E_{1}(C / 2)\right) f\right|^{2} \geqq C^{2}|f|^{2} .
$$

This implies, if $C_{1}$ is the bound of $T$,

$$
(C / 2)^{2}\left|E_{1}(C / 2) f\right|^{2}+C_{1}^{2}\left|\left(1-E_{1}(C / 2)\right) f\right|^{2} \geqq C^{2}|f|^{2} .
$$

Using $\left|E_{1}(C / 2) f\right|^{2}=|f|^{2}-\left|\left(1-E_{1}(C / 2)\right) f\right|^{2}$, we get

$$
\left(C_{1}^{2}-C^{2} / 4\right)\left|\left(1-E_{1}(C / 2)\right) f\right|^{2} \geqq(3 / 4) C^{2}|f|^{2} .
$$

Thus there exists a constant $K$ such that $\left|\left(1-E_{1}(C / 2)\right) f\right|^{2} \geqq K^{2}|f|^{2}$ for all $f$ in $\mathfrak{M}$. Since $1-E(C / 2)$ is bounded this establishes the result. 
Let $B$ be the contraction of $1-E_{1}(C / 2)$ with domain $\mathfrak{M}$. From the above we see that $B^{-1}$ exists and has bound $K^{-1} . B$ is closed since it is defined on a closed linear manifold. Since $B^{-1}$ exists and is bounded $\overline{\mathfrak{M}}=\Re_{B}$ is also closed. $\overline{\mathfrak{M}} \subset \Re_{1-E(C / 2)}, \subset \Re_{1-E_{1}(0)}$, and $\overline{\mathfrak{M}}^{\perp} \supset \mathfrak{N}_{T}$.

Lemma 4. $T E_{1}\left(\lambda_{0}\right) T^{\prime \prime}$ and $T\left(1-E_{1}\left(\lambda_{0}\right)\right) T^{\prime \prime}$ have bound 1.

We have $T E_{1}\left(\lambda_{0}\right)=E_{2}\left(\lambda_{0}\right) T$ and $T\left(1-E_{1}\left(\lambda_{0}\right)\right)=\left(1-E_{2}\left(\lambda_{0}\right)\right) T$ (cf. $\left[8, \S 5\right.$, Definition 1, p. 312 and Theorem IV, p. 313]). Applying $T^{\prime \prime}$ first we get $T E_{1}\left(\lambda_{0}\right) T^{\prime \prime} \subset E_{2}\left(\lambda_{0}\right)$ and $T\left(1-E_{1}\left(\lambda_{0}\right)\right) T^{\prime \prime} C 1-E_{2}\left(\lambda_{0}\right)$ and hence $T E_{1}\left(\lambda_{0}\right) T^{\prime \prime}$ and $T\left(1-E_{1}\left(\lambda_{0}\right)\right) T^{\prime \prime}$ have bound 1 .

LEMmA 5. If $\overline{\mathfrak{M}}=\mathfrak{R}_{\bar{E}}$ is included in the range of $1-E_{1}\left(\lambda_{0}\right), \lambda_{0}>0$, then $T \bar{E} T^{\prime \prime}$ is bounded.

If $\bar{E}$ is the orthogonal projection on $\overline{\mathfrak{M}}, \bar{E}=\bar{E}\left(1-E_{1}\left(\lambda_{0}\right)\right)$. The transform is $T \bar{E} T^{\prime \prime}=T \bar{E}\left(1-E_{1}\left(\lambda_{0}\right)\right) T^{\prime \prime}=T \bar{E}\left(1-E_{1}(0)\right)\left(1-E_{1}\left(\lambda_{0}\right)\right) T^{\prime \prime}$ $=T \bar{E} T^{\prime \prime} T\left(1-E_{1}\left(\lambda_{0}\right)\right) T^{\prime \prime}$. $T$ is an isomorphism on the range of $1-E_{1}\left(\lambda_{0}\right)$ and hence the complementation of $\overline{\mathfrak{M}}$ and $\mathscr{S} \ominus \mathfrak{M}_{1}\left(\lambda_{0}\right) \ominus \overline{\mathfrak{M}}$ relative to $\mathfrak{E} \ominus \mathfrak{M}_{1}\left(\lambda_{0}\right)$ must be preserved as a complementation relation relative to the range of $1-E_{2}\left(\lambda_{0}\right)$. Thus $T \bar{E} T^{\prime \prime}$ is bounded relative to the range of $1-E_{2}\left(\lambda_{0}\right)$ which includes that of $T\left(1-E_{1}\left(\lambda_{0}\right)\right) T^{\prime \prime}$. Since $T\left(1-E_{1}\left(\lambda_{0}\right)\right) T^{\prime \prime}$ is also bounded, $T \bar{E} T^{\prime \prime} T\left(1-E_{1}\left(\lambda_{0}\right)\right) T^{\prime \prime}=T \bar{E} T^{\prime \prime}$ is bounded.

LEMMA 6. $T B^{-1} T^{\prime \prime}$ exists and is bounded.

First we establish that the domain of $T B^{-1} T^{\prime \prime}$ is $T \overline{\mathfrak{M}}$. For $\mathfrak{D}_{T_{B^{-1}} T^{\prime \prime}}$ $=\mathfrak{D}_{B^{-1} T}$. Since $T$ and $T^{\prime \prime}$ are in an inverse relationship relative to $\Re_{1-\bar{E}_{1}(0)}$, which includes $\overline{\mathfrak{M}}, T \overline{\mathfrak{M}}=T \Re_{B}=T \mathfrak{D}_{B^{-1}}=\mathfrak{D}_{B^{-1}} T^{\prime \prime}$.

For $f \in \bar{M} \subset \Re_{1-E_{1}\left(\lambda_{0}\right)}$, we have $|T f| \geqq \lambda_{0}|f|$. Since $T^{\prime \prime}$ and $T$ are inversial for $g \in T \overline{\mathfrak{M}},\left|T^{\prime \prime} g\right| \leqq\left(1 / \lambda_{0}\right)|g|$. Since $B^{-1}$ and $T$ are bounded this yields the desired result.

LEMMA 7. $B^{-1} \bar{E}$ is a projection with range $\mathfrak{M}$ and manifold of zeros $\overline{\mathfrak{M}}^{\perp}$.

Since $\bar{E} \leqq 1-E_{1}\left(\lambda_{0}\right)$ and $B C 1-E_{1}\left(\lambda_{0}\right)$, we have $\bar{E} B^{-1} g=g$ for $g$ in $\Re_{\bar{E}}=\overline{\mathfrak{M}}=\Re_{B}=\mathfrak{D}_{B^{-1}}$. Thus $\bar{E} B^{-1} C 1$ and $\left(B^{-1} \bar{E}\right)\left(B^{-1} \bar{E}\right)=B^{-1} \bar{E} . B^{-1} \bar{E}$ is bounded and has domain the full space since $\Re_{\bar{E}}=\mathfrak{D}_{B^{-1}}$. Hence it is a projection. Since $\Re_{\bar{E}}=\mathfrak{D}_{B^{-1}}, \quad \Re_{B^{-1} \bar{E}}=\Re_{B^{-1}}=\mathfrak{D}_{B}=\mathfrak{M} . \quad \mathfrak{R}_{B^{-1} \bar{E}}=\mathfrak{N}_{\bar{E}}$ $=\overline{\mathfrak{M}}^{\perp}$.

We are now in a position to obtain our result. For $T B^{-1} \bar{E} T^{\prime \prime}$ $=T B^{-1}\left(1-E_{1}(0)\right) \bar{E} T^{\prime \prime}=T B^{-1} T^{\prime \prime} T \bar{E} T^{\prime \prime}=\left(T B^{-1} T^{\prime \prime}\right)\left(T \bar{E} T^{\prime \prime}\right)$ which is bounded by Lemmas 5 and 6 . Since $\overline{\mathfrak{M}}^{\perp} \supset \mathfrak{N}_{T}$, Lemma 2 shows that $T$ preserves the complementation of $\mathfrak{M}$ and $\overline{\mathfrak{M}}^{\perp}$. 


\section{Appendix IV}

We give an example in Hilbert space of a closed $T$, whose domain includes a cl. $1 \mathfrak{M}$, for which $T_{\mathfrak{M}}$ is not closed nor has a closed extension. We first prove the following lemma.

LEMmA. Let $\left\{\left\{a_{1}^{(k)}, a_{2}^{(k)}, \cdots\right\}\right\}$ denote a sequence of sequences with the property that $\sum_{i=1}^{\infty}\left|a_{1}^{(k)}\right|^{2} \rightarrow 0$, as $k \rightarrow \infty$, and for a $K$ independent of $k, \sum_{i=1}^{\infty}\left|a_{i}^{(k)} i 2^{i}\right|^{2}<K$. Then $\sum_{i=1}^{\infty}\left|a_{i}^{(k)}\right| i \rightarrow 0$, as $k \rightarrow \infty$.

Proof. No clarity is lost if we suppress $k$. Let $c_{i}=a_{i} i 2^{i}$. By hypothesis, $\sum_{i=1}^{\infty}\left|c_{i}\right|^{2}<K$. Now $\sum_{i=N+1}^{\infty} i\left|a_{i}\right|=\sum_{i=N+1}^{\infty} 2^{-i}\left|c_{i}\right|<K 2^{-N} 3^{1 / 2}$, by Schwarz's inequality. Our hypotheses also imply that for every $N, \sum_{i=1}^{N}\left|a_{i}\right| i \rightarrow 0$. These results imply that $\lim \sup \sum_{i=1}^{\infty}\left|a_{i}\right| \cdot i$ $<K 2^{-N} 3^{1 / 2}$ for every $N$. Hence $\sum_{i=1}^{\infty}\left|a_{i}\right| i \rightarrow 0$.

Let $\phi_{0}, \phi_{1}, \cdots$ be a complete orthonormal set in $\mathfrak{S}$, let $\psi_{k}=\phi_{2 k-1}$, for $k=1,2, \cdots$ and $\omega_{k}=\phi_{2 k-2}$ for $k=1,2, \cdots$. We define a transformation $T^{\prime \prime}$ by the equations $T^{\prime \prime} \psi_{k}=\phi_{k}, T^{\prime \prime} \omega_{k}=-k 2^{k} \phi_{k}+k \phi_{0}$, $k=1,2, \cdots . T^{\prime \prime}$ has a linear extension $T^{\prime} . f$ is in the domain of $T^{\prime}$, if $f=\sum_{i=1}^{n} a_{i} \omega_{i}+\sum_{i=1}^{n} b_{i} \psi_{k}$ and $T^{\prime} f=\left(\sum_{i=1}^{n} i a_{i}\right) \phi_{0}+\sum_{i=1}^{n}\left(b_{i}-i a_{i} 2^{i}\right) \phi_{i}$.

The condition that $T^{\prime}$ have a closed extension $T$ is that whenever $\left\{f^{(k)}\right\}$ is a sequence in the domain of $T^{\prime}$ such that $\left|f^{(k)}\right|^{2} \rightarrow 0$ and $T^{\prime} f^{(k)} \rightarrow g$, then $g=\theta$. Suppose we have such a sequence of $f^{\prime}$ s and let $g=\sum_{i=0}^{\infty} c_{i} \phi_{i}$. We shall show that $g=\theta$.

To do this we first show that $c_{0}=0$. Suppressing the superscript we let $f$ determine $a_{i}$ and $b_{i}$ as above. Then $\sum_{i=1}^{\infty} i a_{i} \rightarrow c_{0}$. Now we have since $f \rightarrow \theta$ and $T f \rightarrow g$, that $\sum_{i=1}^{\infty}\left|a_{i}\right|^{2}+\sum_{i=1}^{\infty}\left|b_{i}\right|^{2} \rightarrow 0$ and that there is a $K$ such that $\sum_{i=1}^{\infty}\left|b_{i}-i a_{i} 2^{i}\right|^{2}<K$. Since the $\sum_{i=1}^{\infty}\left|b_{i}\right|^{2}$ are bounded we may even infer $\sum_{i=1}^{\infty}\left|i a_{i} 2^{i}\right|^{2}<K_{1}$ for a constant $K_{1}$. Our

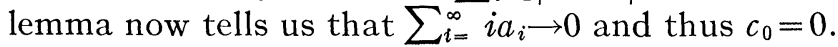

Since for $i \geqq 1$, we have $\left(b_{i}-i 2^{i} a_{i}\right) \rightarrow c_{i}$, we must also have $c_{i}=0$ for $i \geqq 1$. Hence $g=\theta$. Thus $T^{\prime}$ has a closed extension $T$.

If $\mathfrak{M}$ is the closed linear manifold determined by the $\psi_{k}, T \mathfrak{M}$ is the set determined by $\phi_{1}, \phi_{2}, \cdots$. Let $\mathbf{c}_{k}$ denote the class of $(1 / k) \omega_{k}$ relative to $\mathfrak{M}$, which is also the class of $h_{k}=2{ }^{k} \psi_{k}+(1 / k) \omega_{k}$. Now $T h_{k}=T^{\prime} h_{k}=2{ }^{k} \phi_{k}-2{ }^{k} \phi_{k}+\phi_{0}=\phi_{0}$. Hence $T h_{k}=\phi_{0}$. Let $c_{0}$ be the class of $\phi_{0}$ relative to $T \mathfrak{M}$. Then $\left|\mathbf{c}_{k}\right|=1 / k$ and hence $c_{k} \rightarrow \theta$. Also $T_{\mathfrak{M}} c_{k}$ $=\mathbf{c}_{0} \rightarrow \mathbf{c}_{0}$, with $\left|\mathbf{c}_{0}\right|=1$ since $\phi_{0}$ is in $(T \mathfrak{M})^{\perp}$. Hence $T_{\mathfrak{M}}$ does not have a closed extension.

\section{Appendix V}

We suppose that each $\mathfrak{B}_{i}$ is separable, regular $\left(\mathfrak{B}_{i}\right.$ reflexive), and strictly convex. If $T$ is closed and preserves minimality relative to $\mathfrak{M}$, then $T_{\mathfrak{M}}$ is closed. 
For suppose $\left\{c_{i}\right\}$ is a sequence of $\mathfrak{B}_{1} / \mathfrak{M}$ such that $\boldsymbol{c}_{i}$ and $T_{\mathfrak{M}} \boldsymbol{c}_{i}$ are convergent to $c$ and $\mathbf{c}^{\prime}$, respectively. Let $h_{i}$ be the minimal element of $\mathbf{c}_{i}, h$ that of $\mathbf{c}, g$ that of $\mathbf{c}^{\prime}$.

There is a linear functional $G$ defined on $\mathfrak{B}_{1} / \mathfrak{M}$ such that $G(\mathbf{c})$ $=|G| \cdot|\mathbf{c}|$. As in Appendix I, there corresponds an $F \in \mathfrak{M}^{\perp}$ with $|F|=|G|, \quad F(h)=G(\mathbf{c})=|G| \cdot|\mathbf{c}|=|F| \cdot|h|$. Also $F\left(h_{i}\right)=G\left(\mathbf{c}_{i}\right)$ $\rightarrow G(\mathbf{c})=F(h)$.

The $\left|h_{i}\right|=\left|c_{i}\right|$ are bounded. Thus there is a subsequence of the $h_{i}$ which is weakly convergent to an $h^{\prime}$ with $\left|h^{\prime}\right| \leqq \lim \left|h_{i}\right|=\lim \left|c_{i}\right|$ $=|\mathbf{c}|=|h|$ or $\left|h^{\prime}\right| \leqq|h|$. Also $|F| \cdot\left|h^{\prime}\right| \geqq F\left(h^{\prime}\right)=F(h)=|F| \cdot|h|$. It follows that $|h|=\left|h^{\prime}\right|$ and $F\left(h^{\prime}\right)=F(h)$. It can be shown that this implies that $h=h^{\prime}$, if the space is strictly convex. ${ }^{32}$

Thus if $\left\{c_{i}\right\}$ is convergent to $c$, then a subsequence of their minimal elements is weakly convergent to the minimal element of $c$. Thus we can choose a subsequence of the $h_{i}$ 's which converges weakly to $h$ and of these a further subsequence can be chosen so that the $T h_{i}$ approach $g$. Thus $\{h, g\}$ is the weak limit of a subsequence of the $\left\{h_{i}, T h_{i}\right\}$. Since the graph is a closed linear set, it is weakly closed. Thus $\{h, g\}$ is in the graph of $T$ and $T h=g$. Hence $T_{\mathfrak{M C}}$ exists and equals $\mathbf{c}^{\prime}$ and $T_{\mathfrak{M}}$ is closed.

\section{Appendix VI}

We give here an example of a transformation, which is not associated with any absolutely bounded resolution in the sense of Lorch or with any bounded resolution in the sense of $\$ 8$.

For if $T=\int_{-C}^{C} \phi(\lambda) d E(\lambda)$, in Lorch's notation for an absolutely bounded resolution, $E(\lambda)$, then the set $\mathfrak{N}_{T}$ of zeros of $T$ has a projection of bound $K$ on it. This is also true if, in the sense of $\S 8, T$ is associated with a bounded resolution. Thus it will be sufficient for our purposes if we construct a $T$ such that $\mathfrak{N}_{T}$ does not possess a bounded projection.

Let us consider the construction given in [10, p. 152] of a manifold without a projection and in particular the terminology used there.

We regard $l_{p}$ as a sum $\sum \oplus l_{p, 3^{n}}$. Let $F_{n}$ be the projection of $l_{p}$ on the $n$th term in this sum, that is, on the $l_{p, 3^{n}}$. Let $E_{n}$ be the projection of $l_{p, 3^{n}}$ on $\mathfrak{M}_{n}$. Let $\lambda_{n}$ be such that $\lambda_{n}\left(F_{n}-E_{n} F_{n}\right)$ has bound 1 . It can

\footnotetext{
${ }^{32}$ One shows that if $h$ is such that $F(h)=|F| \cdot|h|$ and $g$ is such that $F(g)=0$, then $|h+g| \geqq|g|$. For $|F| \cdot|h+g| \geqq|F(h+g)|=F(h)=|F| \cdot|h|$. Thus $h$ and $h^{\prime}$ are minimal relative to the set of $g^{\prime}$ 's for which $F(g)=0$. Since $F\left(h^{\prime}-h\right)=F\left(h^{\prime}\right)-F(h)=0$, they are in the same class relative to this set. For a strictly convex space this minimum in a class is unique.
} 
readily be shown that $T=\sum_{i=0}^{\infty} \lambda_{n}\left(F_{n}-E_{n} F_{n}\right)$ has bound 1 and that the zeros of $T$ form the set $\mathfrak{B}$, which has no bounded projection on it.

\section{REFERENCES}

1. S. Banach, Théorie des Opérations Lineaires, Warsaw, 1932.

2. H. F. Bohnenblust and A. Sobczyk, this Bulletin, vol. 44 (1938), pp. 91-93.

3. J. Clarkson, Transactions of this Society, vol. 40 (1936), pp. 396-414.

4. S. Kakutani, Japanese Journal of Mathematics, vol. 16 (1939), pp. 93-97.

5. E. R. Lorch, Transactions of this Society, vol. 45 (1939), pp. 217-234.

6. ——, this Bulletin, vol. 45 (1939), pp. 564-569.

7. D. Milman, Comptes Rendus de l'Académie des Sciences de l'URSS (n.s.), vol. 20 (1938), pp. 243-246.

8. F. J. Murray, Transactions of this Society, vol. 37 (1935), pp. 301-338.

9. - Transactions of this Society, vol. 39 (1936), pp. 83-100.

10. - Transactions of this Society, vol. 41 (1937), pp. 138-152.

11. - Annals of Mathematics Studies, Princeton, no. 4 (1941).

12. B. J. Pettis, Duke Mathematical Journal, vol. 5 (1939), pp. 249-253.

13. R. S. Phillips, this Bulletin, vol. 46 (1940), pp. 930-933.

14. A. Sobczyk, Duke Mathematical Journal, vol. 8 (1941), pp. 78-106.

15. M. H. Stone, American Mathematical Society Colloquium Publications, vol. 15 (1932).

16. J. von Neumann, Annals of Mathematics, (2), vol. 33 (1932), pp. 294-310.

Columbia University 